\title{
Occlusal outcome after orthodontic treatment with preadjusted straight-wire and standard edgewise appliances
}

\section{A retrospective cohort study}

\author{
Spyridon N. Papageorgiou' ${ }^{1 D} \cdot$ Raphael Tilen $^{1} \cdot$ Vaska Vandevska-Radunovic $^{2}$ (D) $\cdot$ Theodore Eliades $^{1}$
}

Received: 22 May 2020 / Accepted: 16 October 2020 / Published online: 13 January 2021

(c) The Author(s) 2020

\begin{abstract}
Purpose Orthodontic fixed appliances have been proven to be effective in treating a wide variety of malocclusions, and different types of appliances have emerged during recent decades. However, the comparative effects of different appliances have not been adequately assessed. Thus, the aim was to assess the occlusal outcome of orthodontic treatment with preadjusted straight-wire (SWIRE) and standard edgewise (SEDGE) appliances.

Methods In all, 56 patients (mean age: 13.5 years; $45 \%$ male) receiving extraction-based treatment with either SWIRE or SEDGE appliances were included. Between-group differences in the occlusal outcome assessed with the American Board of Orthodontists Objective Grading System (ABO-OGS) and treatment duration were analyzed statistically at the 5\% level. Results The average ABO-OGS score was $31.3 \pm 7.2$ points and $34.0 \pm 10.4$ points in the SWIRE and SEDGE groups with no statistically significant difference between groups $(P=0.26)$. Treatment duration was significantly shorter in the SWIRE group compared to the SEDGE group, with an average difference of -6.8 months (95\% confidence interval [95\% $\mathrm{CI}]=-9.6$ to -4.0 months; $P<0.001)$. Likewise, fewer visits were needed with SWIRE compared to SEDGE appliances with an average difference of -7.2 visits $(95 \% \mathrm{CI}=-10.3$ to -4.2 visits; $P<0.001)$. Adjusting for the influence of any potential confounders did not considerably impact the results.

Conclusion Similar treatment outcomes were observed after premolar extraction treatment with SWIRE and SEDGE appliances. On the other hand, SEDGE appliances were associated with prolonged treatment duration and more visits needed to complete treatment compared to SWIRE appliances.
\end{abstract}

Keywords Treatment outcome $\cdot$ Treatment duration $\cdot$ Orthodontics $\cdot$ Fixed appliances $\cdot$ Retrospective cohort study

Protocol and registration The protocol was registered prior to the study (ISRCTN ID 13048456) and is publicly assessable (https:// osf.io/e $3 \mathrm{j} 5 \mathrm{f} /$ ).

Availability of data and materials All data generated or analyzed during this study are included in this published article or its supplements, while its dataset is openly provided through Zenodo (https://doi.org/10.5281/zenodo.3663626).

Supplementary Information The online version of this article (https://doi.org/10.1007/s00056-020-00273-z) contains

supplementary material, which is available to authorized users.

Spyridon N. Papageorgiou

snpapage@gmail.com
Raphael Tilen

Raphael.Tilen@zzm.uzh.ch

Vaska Vandevska-Radunovic

vaska.vandevska-radunovich@odont.uio.no

Theodore Eliades

theodore.eliades@zzm.uzh.ch

1 Clinic of Orthodontics and Pediatric Dentistry, Center of Dental Medicine, University of Zurich, Plattenstraße 11, Zurich, Switzerland

2 Department of Orthodontics, Faculty of Dentistry, University of Oslo, Oslo, Norway 


\section{Okklusales Ergebnis nach kieferorthopädischer Behandlung mit "straight-wire“- und „standard edgewise"-Apparaturen}

Eine retrospektive Kohortenstudie

\section{Zusammenfassung}

Zielsetzung Festsitzende kieferorthopädische Apparaturen haben sich bei der Behandlung einer Vielzahl von Fehlstellungen als wirksam erwiesen, und in den letzten Jahrzehnten sind verschiedene Apparaturtypen entwickelt worden. Die unterschiedlichen Auswirkungen der verschiedenen Apparaturen sind jedoch noch nicht angemessen erforscht worden. Ziel war es daher, das okklusale Outcome nach einer kieferorthopädischen Behandlung mit vorjustierten SWIRE(,straight-wire“)und SEDGE(,,standard edgewise“)-Apparaturen zu untersuchen.

Methoden Insgesamt wurden 56 Patienten (Durchschnittsalter 13,5 Jahre, 45\% männlich) aufgenommen, die eine extraktionsbasierte Behandlung entweder mit SWIRE- oder SEDGE-Geräten erhielten. Die Unterschiede zwischen den Gruppen hinsichtlich des anhand des ABO-OGS (American Board of Orthodontists Objective Grading System) bewerteten okklusalen Outcomes und der Behandlungsdauer wurden auf der 5\%-Ebene statistisch analysiert.

Ergebnisse Der durchschnittliche ABO-OGS-Score betrug 31,3 \pm 7,2 Punkte in der SWIRE- und 34,0 $\pm 10,4$ Punkte in der SEDGE-Gruppe und es gab keinen statistisch signifikanten Unterschied zwischen den Gruppen $(P=0,26)$. Die Behandlungsdauer war in der SWIRE-Gruppe im Vergleich zur SEDGE-Gruppe mit einem durchschnittlichen Unterschied von -6,8 Monaten (95\% Konfidenzintervall [95\%-KI] -9,6 bis -4,0 Monate; $P<0,001)$ signifikant kürzer. Entsprechend waren mit SWIRE weniger Kontrolluntersuchungen erforderlich als mit SEDGE-Geräten, mit einer durchschnittlichen Differenz von -7,2 (95\%-KI -10,3 bis -4,2; $P<0,001)$. Die Anpassung unter Berücksichtigung des Einflusses möglicher Confounder hatte keinen wesentlichen Einfluss auf die Ergebnisse.

Schlussfolgerung Ähnliche Behandlungsergebnisse wurden nach Prämolarenextraktionsbehandlung mit SWIRE- und mit SEDGE-Apparaturen beobachtet. Auf der anderen Seite waren bei der Verwendung von SEDGE-Apparaturen im Vergleich zu SWIRE-Apparaturen eine längere Behandlungsdauer und mehr Besuche erforderlich, um die Behandlung abzuschließen.

Schlüsselwörter Ergebnis der Behandlung · Behandlungsdauer · Kieferorthopädie · Festsitzende Apparaturen · Retrospektive Kohortenstudie

$\begin{array}{ll}\text { Abbreviations } \\ \text { ABO } & \text { American Board of Orthodontics } \\ \text { ALARA } & \text { as low as reasonably achievable } \\ \text { CI } & \text { Confidence interval } \\ \text { ICON } & \text { Index of Complexity Outcome and Need } \\ \text { IQR } & \text { interquartile range } \\ \text { OGS } & \text { Objective Grading System } \\ \text { SD } & \text { standard deviation } \\ \text { SEDGE } & \text { standard edgewise } \\ \text { STROBE } & \text { Strengthening the Reporting of Observational } \\ & \text { Studies in Epidemiology } \\ \text { SWIRE } & \text { straight-wire }\end{array}$

\section{Introduction}

Reliable assessment of patient records after treatment with a reliable tool is important in measuring treatment success in an objective way. Multiple indices have been introduced that make case evaluation easier [1-5] and although the Peer Assessment Rating tool [4] is one of the most widely used, it does mostly measure malocclusion improvement and not precisely measure tooth positions within the occlusion. The Index of Complexity Outcome and Need (ICON)
[5] was ambitiously developed to easily evaluate case complexity, treatment need, and malocclusion improvement in an objective way, but its esthetical component might receive disproportionate weight for assessments of treatment outcome.

A more efficient method to assess outcome of orthodontic treatment might be the Objective Grading System (OGS) from the American Board of Orthodontics (ABO) [6] that uses cast models and orthopantomograms after debonding. The ABO-OGS tool gauges finishing quality of the final occlusion based on eight criteria reflecting ideal intercuspation and function. Ideal arch alignment and intercuspation are scored with 0 points, deviations from ideal for each criterion are scored with 1-2 penalty points per tooth and a case can ultimately be categorized as "success" or "failure"- - a system that shows high accordance in both inter- and intraexaminer level. The ABO-OGS has been extensively used in recent years - to compare several orthodontic treatment models and techniques [7-16] with enhanced reliability, validity, and precision when evaluating the progress or final outcome of fixed appliance treatment $[17,18]$. Furthermore, clinical data indicated that treatment outcomes fulfilling the standards of the ABO-OGS led to a balanced anterior temporalis activation and improved chewing function as re- 
ported by patients [19]. Finally, ABO-OGS is able to detect fine changes that occur in the occlusion postdebonding [20, $21]$ and evidence supports that better finishing quality with ABO-OGS leads to better long-term outcomes [22].

Fixed appliance treatment has become an integral part of modern orthodontics and has been a major focus point of orthodontic research. Since their initial introduction by Edward H. Angle, considerable development has been seen, with appliances becoming more and more sophisticated. A major historical point was the development of the preadjusted appliance by L. Andrews [2], which was followed by several variations in the values for tip and torque prescribed for each tooth. In recent years, focus has been added to try to make orthodontic treatment as efficient as possible, so as to minimize adverse effects and maximize patient satisfaction [23]. A recent systematic review on various orthodontic fixed appliances [24] concluded that at the present there is very little evidence from controlled trials to form clinical recommendations about their comparative efficacy. Comparisons between preprogrammed and nonprogrammed appliances are limited and, in their majority, suffer from methodological limitations of uncontrolled studies including confounding and assessor bias.

The aim of this study is therefore to compare the results of orthodontic treatment with preadjusted straightwire (SWIRE) and nonprogrammed standard edgewise (SEDGE) appliances. The primary research question is whether SWIRE appliances result in better occlusal outcome measured by ABO-OGS after class II extraction treatment compared to SEDGE appliances.

\section{Materials and methods}

\section{Protocol, registration, and ethical approval}

The protocol for this study was developed a priori, registered in the ISRCTN registry (ID 13048456), is openly available in the Open Science Framework (https://osf.io/ e $3 \mathrm{j} 5 \mathrm{f} /$ ), and deviations from it were noted (Appendix 1). Ethical approval was received from the ethical institutional authorities of the University of Zurich (BASEC no.: 2018-00631) and the University of Oslo (Regional Committees for Medical and Health Research Ethics; ref. no.: 2017/1885). This report is based on the Strengthening the Reporting of Observational Studies in Epidemiology (STROBE) statement [25].

\section{Sample}

Included in this retrospective parallel multicenter cohort study were patients seeking comprehensive orthodontic treatment with fixed appliances with premolar extractions in two postgraduate university clinics in Oslo (Norway) and Zurich (Switzerland). As a standard procedure, informed consent was acquired from all patients or their guardians before treatment. Eligible patients should comply with the following patient-related inclusion criteria: (i) any ethnicity or race; (ii) male or female; (iii) class I, class II, or class III malocclusion; (iv) full complement of teeth excluding the third molars; (v) no previous orthodontic treatment; (vi) no dentofacial deformities or clefts; and (vii) complete set of pretreatment and posttreatment records. Additionally, they should comply with the following treatment-related inclusion criteria: (i) one phase treatment with labial fixed appliances in both arches (no two-phase treatment); (ii) bilateral extraction of a premolar in one or two jaws (either 2- or 4-premolars extracted); (iii) no temporary anchorage devices of any form; (iv) no orthognathic surgery; (v) no dental trauma; and (vi) no impacted canines. Patients from the two university clinics were selected randomly from patients treated in the last 10 years that fulfilled the eligibility criteria without consideration of pretreatment characteristics, treatment outcome, or treatment duration.

Treatment planning and treatment procedures followed each institution's typical workflow, where all patients are treated by dentists undergoing specialty training in orthodontics under the direct supervision of university faculty with extensive clinical experience. Conventionally ligated labial fixed appliances were used in all cases with the only difference that one clinic (University of Oslo) used SWIRE appliances (MBT Victory, 3M Unitek, Monrovia, CA, USA) and the other clinic (University of Zurich) used SEDGE appliances (Mini Twin Diamond, Ormco, Orange, CA, USA). Contrary to the University of Oslo, in the University of Zurich patients are primarily treated in most cases with SEDGE appliances and only a handful of selected simple cases of each postgraduate student (less than $3 \%$ ) are treated with SWIRE appliances. Both appliance systems had a 0.018-inch slot and the archwire sequence/ mechanics were left to the discretion of the experienced clinical instructors supervising treatment.

The records used in this study were pre- and posttreatment plaster dental casts, panoramic x-rays, and lateral cephalometric $\mathrm{x}$-rays. These records had already been taken within the normal course of orthodontic treatment after consent of the patient for diagnostic reasons, according to the principles of evidence-based medicine and the as low as reasonably achievable (ALARA) principle. All lateral cephalometric $\mathrm{x}$-rays were taken in natural head position and were analyzed using appropriate orthodontic analysis methods.

From each patient's documentation the following pretreatment data were extracted: age, sex, overjet, overbite, ANB angle, and SN-ML angle. In addition, the pretreatment Discrepancy Index (DI), a pretreatment scoring system developed by the $\mathrm{ABO}$ for phase III of the orthodontic board 
certification exam, was calculated. The DI has become an accepted and reliable index for quantifying the complexity of cases based on pretreatment orthodontic record analysis and measurements from dental casts and radiographs $[26,27]$. Finally, it was noted whether (i) 4 (instead of 2) premolars were extracted, (ii) a transpalatal arch was used, and (iii) a headgear was used in conjunction with the fixed appliances.

\section{Sample size calculation}

A priori sample size calculation for the primary outcome of ABO OGS was done based on the previous study of Mislik et al. [28] using: (i) control mean of 25.7 points, (ii) standard deviation of 8.7 points-assumed common between groups, (iii) a clinically meaningful difference in ABO OGS of $30 \%$ of the control mean, (iv) use of an unpaired Student's t-test, (v) alpha of 5\%, and (vi) beta of $20 \%$. With these baseline data and assumptions, a needed sample of 22 patients/group (for a total of 44 patients) was calculated. In order to account for patient losses due to poorquality radiographs/models this was rounded up by $25 \%$ to 28 patients/group (to a total of 56 patients).

\section{Outcomes}

The primary outcome of this study was the total ABO-OGS score after debonding, measured from the patient's posttreatment plaster models and orthopantomograms. All eight ABO-OGS components were evaluated with the special $\mathrm{ABO}$ gauge: alignment, marginal ridges, buccolingual inclination, overjet, occlusal contacts, occlusal relationships, interproximal contacts, and root angulation. The principal investigator (SNP) and a second author (RT) had prior to the study completed the necessary calibration process as instructed by the $\mathrm{ABO}$ and were calibrated with ten random cases not included in this study. The overall cumulative score for all ABO-OGS categories was used as primary outcome. The secondary outcomes included (a) the score of each separate ABO-OGS category, (b) treatment duration in months, and (c) number of visits. No blinding considering treatment decisions and procedures could have been undertaken. However, all study material was anonymized and subsequently scored, so that outcome measurement and statistical analysis could be performed in a blinded manner.

\section{Statistical analysis}

Normality was checked through visual graph inspection and formally with the Shapiro-Wilk test. Descriptive statistics were calculated including means with standard deviations (SDs) for normal data or medians with interquartile ranges (IQRs) for non-normal data. Differences between groups were assessed with Student's t-tests for independent samples or Mann-Whitney tests, accordingly. Crude linear regression modelling was used to assess the effect of appliance on the primary or secondary outcome (total ABO-OGS score and treatment duration, respectively) and its $95 \%$ confidence intervals (CIs). Adjusted analyses were done using the change-in-estimate method to select potential confounders with a minimum of $10 \%$ change set as cut-off [29]. A sample of 25 patients was randomly chosen and measured by both the first (SNP) and the second author (RT), while another random sample of 25 patients was remeasured by the first assessor (SNP) after 2 weeks for repeatability. Repeatability and agreement of the measurements were assessed with the concordance correlation coefficient [30] and the Bland-Altman method [31]. Alpha was set at a two-sided $P<0.05$, all analyses were done in Stata SE 14.2 (StataCorp, College Station, TX, USA), and the data set was openly provided [32].

\section{Results}

A total of 56 patients were included in this study, who were treated either with SWIRE $(n=28)$ or with SEDGE appliances $(n=28)$. No significant differences existed for most characteristics (Table 1). Among the included patients $25(45 \%)$ were male, the mean age was 13.5 years $(\mathrm{SD}=2.4$ years $)$, the mean overjet $5.0 \mathrm{~mm}(\mathrm{SD}=2.2 \mathrm{~mm})$, the mean overbite $3.1 \mathrm{~mm}(\mathrm{SD}=2.4 \mathrm{~mm})$, the mean $\mathrm{ANB}$ $3.9^{\circ}\left(\mathrm{SD}=2.2^{\circ}\right)$, and the mean SN-ML $36.2^{\circ}\left(\mathrm{SD}=5.6^{\circ}\right)$. A total of 40 patients $(71 \%)$ were treated with 4 premolar extractions and 43 patients $(77 \%)$ received a transpalatal arch. The only statistically significant difference between groups was the use of headgear, where more SWIRE pa-

Table 1 Characteristics of patients included in the study Tab. 1 Merkmale der in die Studie aufgenommenen Patienten

\begin{tabular}{llll}
\hline & SWIRE & SEDGE & $P$ \\
\hline Patients, $n$ & 28 & 28 & - \\
Male, $n(\%)$ & $14(50 \%)$ & $11(39 \%)$ & $0.42^{\mathrm{a}}$ \\
Age, mean (SD) & $13.2(1.3)$ & $13.7(3.2)$ & $0.45^{\mathrm{b}}$ \\
Overjet, mean (SD) & $5.1(2.5)$ & $4.8(1.8)$ & $0.60^{\mathrm{b}}$ \\
Overbite, mean (SD) & $2.5(3.0)$ & $3.8(1.4)$ & $0.05^{\mathrm{b}}$ \\
ANB, mean (SD) & $4.1(2.3)$ & $3.8(2.2)$ & $0.60^{\mathrm{b}}$ \\
SN-ML, mean (SD) & $36.8(5.7)$ & $35.6(5.4)$ & $0.41^{\mathrm{b}}$ \\
4-premolar extraction, $n(\%)$ & $20(71 \%)$ & $20(71 \%)$ & $1.00^{\mathrm{a}}$ \\
TPA, $n(\%)$ & $13(46 \%)$ & $8(29 \%)$ & $0.17^{\mathrm{a}}$ \\
Headgear, $n(\%)$ & $25(89 \%)$ & $18(64 \%)$ & $\mathbf{0 . 0 3}^{\mathrm{a}}$ \\
\hline
\end{tabular}

$S D$ standard deviation, SEDGE standard edgewise group, SWIRE straight-wire group, TPA transpalatal arch (and/or lingual arch)

${ }^{\text {a }}$ From chi square test

${ }^{\mathrm{b}}$ From t-test for independent samples 
Table 2 American Board of Orthodontists' Discrepancy Index in straight-wire (SWIRE) and standard edgewise (SEDGE) groups

Tab. 2 Diskrepanzindex des American Board of Orthodontists in der SWIRE(,straight-wire“)- und der SEDGE(,standard edgewise“)-Gruppe

\begin{tabular}{|c|c|c|c|c|c|}
\hline \multirow[b]{2}{*}{ DI component } & \multicolumn{2}{|l|}{ SWIRE } & \multicolumn{2}{|l|}{ SEDGE } & \multirow[t]{2}{*}{$P^{\mathrm{a}}$} \\
\hline & Median (IQR) & Range & Median (IQR) & Range & \\
\hline DI category: overjet & $3(2-4)$ & $0-8$ & $3(3-4)$ & $1-7$ & 0.45 \\
\hline DI category: overbite & $0(0-2)$ & $0-5$ & $2(0-3)$ & $0-3$ & 0.25 \\
\hline DI category: anterior open bite & $1.5(0-4.5)$ & $0-37$ & $0(0-0)$ & $0-4$ & $<0.001$ \\
\hline DI category: lateral open bite & $0(0-0)$ & $0-24$ & $0(0-0)$ & $0-6$ & 0.25 \\
\hline DI category: crowding & $4(2-7)$ & $0-7$ & $2(1-7)$ & $0-7$ & 0.04 \\
\hline DI category: occlusal relationship & $4(0-5)$ & $0-8$ & $4(1-4.5)$ & $0-8$ & 0.93 \\
\hline DI category: lingual posterior crossbite & $0(0-0)$ & $0-2$ & $0(0-0)$ & $0-4$ & 0.79 \\
\hline DI category: buccal posterior crossbite & $0(0-0)$ & $0-4$ & $0(0-0)$ & $0-6$ & 0.99 \\
\hline DI category: cephalometrics & $3(0-11)$ & $0-32$ & $4(0-8)$ & $0-21$ & 0.71 \\
\hline DI category: other & $2(0-3)$ & $0-6$ & $2(0-2)$ & $0-8$ & 0.91 \\
\hline DI total score & $22.5(14.5-31)$ & $6-104$ & $19.5(15-23)$ & $11-41$ & 0.26 \\
\hline
\end{tabular}

$D I$ discrepancy index, $I Q R$ interquartile range

${ }^{a}$ From Mann-Whitney test

tients received a headgear than SEDGE patients $(89 \%$ versus $64 \%$, respectively; $P=0.03$ ).

Analysis with the DI (Table 2) showed that the two groups were on average similar in terms of baseline malocclusion severity $(P=0.26)$. The only significant differences between the two groups pertained to the DI criteria of anterior open bite and crowding. On average, patients in the SWIRE groups had significantly more anterior open bite (medians of 1.5 and 0 , respectively; $P<0.001$ ) and more crowding than SEDGE patients (medians of 4 and 2, respectively; $P=0.04$ ).

The total ABO-OGS score was similar in the SWIRE and SEDGE groups (means of 31.3 and 34.0 points, respectively) with no statistically significant difference $(P=0.26$;
Table 3, Fig. 1a). Similar scores were also seen for most separate ABO-OGS criteria $(P>0.05)$ with the sole exception of overjet, where SWIRE patients had significantly better alignment than SEDGE patients (means of 3.9 and 5.4 points, respectively; $P=0.03$ ). The treatment duration was significantly lower in the SWIRE group compared to the SEDGE group (means of 25.7 and 32.5 months, respectively; $P<0.001$; Fig. 1b). This translated to an average difference of -6.8 months ( $95 \% \mathrm{CI}=-9.6$ to -4.0 months) between groups. The number of visits was similarly lower in the SWIRE group compared to the SEDGE group (means of 24.5 and 31.5 visits, respectively; $P<0.001$ ) with an average difference of -7.2 visits $(95 \% \mathrm{CI}=-10.3$ to -4.2 visits). This was not due to different interval between the

Table 3 Results of outcomes assessed

Tab. 3 Bewertungen der Behandlungsergebnisse

\begin{tabular}{|c|c|c|c|}
\hline Variable & SWIRE & SEDGE & $P$ \\
\hline Patients, $n$ & 28 & 28 & - \\
\hline ABO Total score, mean (SD) & $31.3(7.2)$ & $34.0(10.4)$ & $0.26^{\mathrm{a}}$ \\
\hline ABO Alignment/rotations, mean (SD) & $7.5(2.4)$ & $9.1(3.8)$ & $0.06^{\mathrm{a}}$ \\
\hline ABO Marginal ridges, mean (SD) & $3.7(1.6)$ & $4.4(2.4)$ & $0.19^{\mathrm{a}}$ \\
\hline ABO Buccolingual inclination, mean (SD) & $4.1(2.8)$ & $3.3(2.3)$ & $0.23^{\mathrm{a}}$ \\
\hline $\mathrm{ABO}$ Overjet, mean (SD) & $3.9(2.3)$ & $5.4(2.9)$ & $\mathbf{0 . 0 3}^{\mathrm{a}}$ \\
\hline ABO Occlusal contacts, median (IQR) & $5.0(3.5-6.0)$ & $6.0(3.0-8.0)$ & $0.35^{\mathrm{b}}$ \\
\hline ABO Occlusal relationships, mean (SD) & $4.3(2.0)$ & $4.0(2.4)$ & $0.68^{\mathrm{a}}$ \\
\hline ABO Interproximal contacts, median (IQR) & $0(0-0)$ & $0(0-0)$ & $0.16^{\mathrm{b}}$ \\
\hline ABO Root angulation, mean (SD) & $2.3(1.5)$ & $1.6(1.2)$ & $0.06^{\mathrm{a}}$ \\
\hline Treatment duration (months), mean (SD) & $25.7(3.5)$ & $32.5(6.6)$ & $<0.001^{\text {a }}$ \\
\hline Number of visits, mean (SD) & $24.5(4.1)$ & $31.5(6.8)$ & $<0.001^{\mathrm{a}}$ \\
\hline Interval between visits (weeks), mean (SD) & $4.8(0.5)$ & $4.5(0.7)$ & $0.11^{\mathrm{a}}$ \\
\hline
\end{tabular}

$I Q R$ interquartile range, $S D$ standard deviation, $S E D G E$ standard edgewise group, SWIRE straight-wire group, ABO American Board of Orthodontists

${ }^{\text {a }}$ From t-test for independent samples

${ }^{\mathrm{b}}$ From Mann-Whitney test 


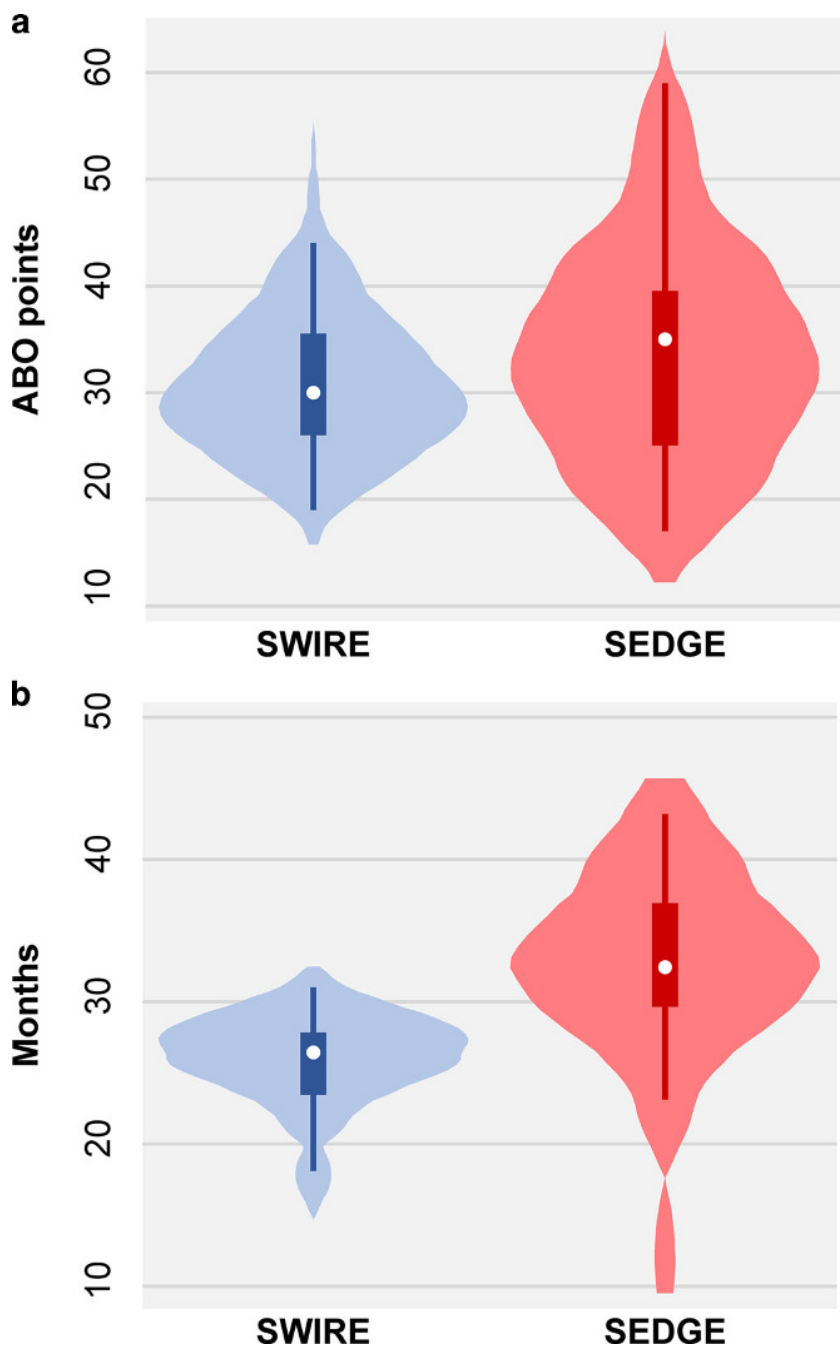

Fig. 1 Violin plot for $\mathbf{a}$ total ABO-OGS score and $\mathbf{b}$ treatment duration in the two groups. $A B O$ American Board of Orthodontics, OGS Objective Grading System

Abb. 1 Violin-Diagramm für a den globalen ABO-OGS-Score, $\mathbf{b}$ Behandlungsdauer in den beiden Gruppen. $A B O$ American Board of Orthodontics, $O G S$ Objective Grading System

visits (means of 4.8 and 4.5 weeks, respectively; $P=0.11$; compared post hoc). Finally, analyses adjusted for the influence of any potential confounders (Appendix 2) found no considerable differences from the main analysis.

Intraexaminer agreement and repeatability was almost perfect with a concordance correlation coefficient of 0.98 (95\% CI $=0.96-0.99)$ and a Bland-Altman average difference of -0.52 (95\% limits of agreement $=-4.08$ to 3.05 ). Interexaminer agreement and repeatability was somewhat worse with a concordance correlation coefficient of 0.87 $(95 \% \mathrm{CI}=0.79-0.92)$ and a Bland-Altman average difference of $-0.82(95 \%$ limits of agreement $=-9.52$ to 7.87$)$.

\section{Discussion}

The current study assessed the occlusal outcome of 56 patients treated with premolar extractions and either SWIRE or SEDGE fixed orthodontic appliances. The main finding of this study was that the appliance type was not significantly associated with the occlusal outcome of treatment according to the total ABO-OGS score $(P=0.26)$. Small differences were seen formally only for the ABO-OGS criterion of overjet, which favored the SWIRE. It is important here to note that 'overjet' in the ABO-OGS pertains not to a single measurement between the upper and lower central incisors, but rather assesses the anteroposterior relationship of every pair of antagonistic anterior teeth and the buccolingual relationship of every pair of antagonistic posterior teeth. As such, differences in this criterion might reflect sagittal discrepancies of the upper to lower dentition or transverse discrepancies of the posterior tooth segments. In addition, penalties in the 'overjet' ABO-OGS criterion might reflect deviations from the ideal inclination or torque of any anterior or posterior tooth, which preclude proper relationships between antagonists.

On the other hand, treatment with SWIRE appliances was found to be more efficient than with SEDGE appliances in terms of shorter duration and fewer visits. This agrees with a previous retrospective study on extraction treatment that reported shorter treatment durations with SWIRE appliances [33]. A possible explanation for this might be that during space closure in the SWIRE group the already attained built-in prescription of the appliance for each tooth is retained to some degree. On the contrary, torque lost during space closure in the SEDGE group might need to be reapplied at the finishing stage, which might result in prolonged treatment times.

The problematic control of tooth inclination/torque during treatment is supported by another retrospective study of nonextraction treatment by Soltani et al. [34], where SWIRE treatment was associated with significantly improved buccolingual inclination of teeth compared to SEDGE treatment. However, a much smaller gain of 2 months in total was seen for the SWIRE group [34], but this might be due to nonextraction treatment and the lack of space closure. This difference with respect to the control of tooth inclination is corroborated by another retrospective study of extraction treatment, where SWIRE patients scored significantly better for the angulation and inclination of the maxillary posterior teeth compared to SEDGE patients [33]. Another retrospective study found significant differences in anchorage loss of the lower incisors between treatment with SWIRE and SEDGE appliances [35], which might lead to longer treatment times needed to upright proclined incisors. Finally, another study found that patients treated with SEDGE appliances did not exhibit 
ideal functional occlusal relationships, whereas most individuals after SWIRE appliance therapy had an ideal (i.e., mutually protected) occlusion [36]. On the other hand, an unpublished retrospective study of extraction/nonextraction cases found no statistically significant difference in either treatment duration or PAR scores between SWIRE and SEDGE appliances [37].

Although root resorption was not measured in this study, a previous retrospective study [38] reported that extraction treatment with SEDGE was associated with significantly greater resorption of the upper incisors compared to the SWIRE group. This might be attributed to more efficient force control in the SWIRE group, where tooth inclination and torque is gradually applied on the teeth already through the aligning superelastic archwires of increasing size and stiffness, whereas these are applied abruptly during the finishing stage in the SEDGE group. Finally, a previous randomized trial did not find a significant difference in either root resorption of the upper incisors or treatment duration between fully programmed (Roth) and partly programmed fixed appliances [39]. However, no pure nonprogrammed SEDGE appliance had been used in this trial, while both extraction and nonextraction cases were pooled together and this might have influenced the results.

The strengths of this retrospective observational study are its a priori registered protocol [40] and its open data provision that increases its transparency [41]. The performed sample size calculation means that the study was adequately powered to identify clinically relevant differences between appliances, while blinded outcome assessment reduces the potential for bias. However, the present study also has certain limitations that need to be considered. First, it was a nonrandomized retrospective study, which means it is more prone to bias than both prospective nonrandomized and randomized studies [42]. Furthermore, as most clinicians pick one appliance prescription and stick to it, it was not feasible to have both SWIRE and SEDGE appliances being administered in the same clinic. Thus, two different centers were compared. Pretreatment case severity was measured with the ABO DI in order to assess and control for baseline differences, but this is no panacea. Finally, important adverse effects like root resorption and gingival recessions were not assessed in this study, even though these are often taken into consideration during clinical decisionmaking regarding implemented appliances and techniques.

\section{Conclusions}

The results of the present retrospective study within its limitations indicate that on average similar occlusal outcomes according to the ABO-OGS are possible after extractionbased orthodontic treatment with the use of either straight- wire or standard edgewise appliances. However, treatment with standard edgewise appliances might take longer on average than treatment with straight-wire appliances. These findings should be confirmed by future prospective clinical studies comparing these two appliances, preferably with a randomized trial design.

Funding Open access funding provided by University of Zurich

\section{Compliance with ethical guidelines}

Conflict of interest S.N. Papageorgiou, R. Tilen, V. VandevskaRadunovic and T. Eliades declare that they have no competing interests.

Ethical standards Ethical approval was received from the ethical institutional authorities of the University of Zurich (BASEC no.: 201800631) and the University of Oslo (Regional Committees for Medical and Health Research Ethics; ref. no.: 2017/1885). As a standard procedure, written informed consent was acquired from all patients or their guardians before treatment.

Open Access This article is licensed under a Creative Commons Attribution 4.0 International License, which permits use, sharing, adaptation, distribution and reproduction in any medium or format, as long as you give appropriate credit to the original author(s) and the source, provide a link to the Creative Commons licence, and indicate if changes were made. The images or other third party material in this article are included in the article's Creative Commons licence, unless indicated otherwise in a credit line to the material. If material is not included in the article's Creative Commons licence and your intended use is not permitted by statutory regulation or exceeds the permitted use, you will need to obtain permission directly from the copyright holder. To view a copy of this licence, visit http://creativecommons.org/licenses/by/4. $0 /$.

\section{References}

1. Summers CJ (1971) The occlusal index: a system for identifying and scoring occlusal disorders. Am J Orthod 59:552-567

2. Andrews LF (1972) The six keys to normal occlusion. Am J Orthod 62:296-309

3. Pickering EA, Vig P (1975) The occlusal index used to assess orthodontic treatment. Br J Orthod 2:47-51

4. Richmond S, Shaw WC, Roberts CT, Andrews M (1992) The PAR Index (Peer Assessment Rating): methods to determine outcome of orthodontic treatment in terms of improvement and standards. Eur J Orthod 14:180-187

5. Daniels C, Richmond S (2000) The development of the index of complexity, outcome and need (ICON). J Orthod 27:149-162

6. Casko JS, Vaden JL, Kokich VG, Damone J, James RD, Cangialosi TJ et al (1998) Objective grading system for dental casts and panoramic radiographs. American Board of Orthodontics. Am J Orthod Dentofacial Orthop 114:589-599

7. Hsieh TJ, Pinskaya Y, Roberts WE (2005) Assessment of orthodontic treatment outcomes: early treatment versus late treatment. Angle Orthod 75:162-170

8. Kuncio D, Maganzini A, Shelton C, Freeman K (2007) Invisalign and traditional orthodontic treatment postretention outcomes compared using the American Board of Orthodontics objective grading system. Angle Orthod 77:864-869

9. Okunami TR, Kusnoto B, BeGole E, Evans CA, Sadowsky C, Fadavi S (2007) Assessing the American Board of Orthodontics ob- 
jective grading system: digital vs plaster dental casts. Am J Orthod Dentofacial Orthop 131:51-56

10. Detterline DA, Isikbay SC, Brizendine EJ, Kula KS (2010) Clinical outcomes of 0.018-inch and 0.022-inch bracket slot using the ABO objective grading system. Angle Orthod 80:528-532

11. Chaison ET, Liu X, Tuncay OC (2011) The quality of treatment in the adult orthodontic patient as judged by orthodontists and measured by the objective grading system. Am J Orthod Dentofacial Orthop 139:S69-75

12. Jain M, Varghese J, Mascarenhas R, Mogra S, Shetty S, Dhakar N (2013) Assessment of clinical outcomes of Roth and MBT bracket prescription using the American Board of Orthodontics objective grading system. Contemp Clin Dent 4:307-312

13. Anthopoulou C, Konstantonis D, Makou M (2014) Treatment outcomes after extraction and nonextraction treatment evaluated with the American Board of Orthodontics objective grading system. Am J Orthod Dentofacial Orthop 146:717-723

14. Penning EW, Peerlings RHJ, Govers JDM, Rischen RJ, Zinad K, Bronkhorst EM et al (2017) Orthodontics with customized versus noncustomized appliances: a randomized controlled clinical trial. J Dent Res 96:1498-1504

15. Yildırım K, Saglam-Aydinatay B (2018) Comparative assessment of treatment efficacy and adverse effects during nonextraction orthodontic treatment of Class I malocclusion patients with direct and indirect bonding: A parallel randomized clinical trial. Am J Orthod Dentofacial Orthop 154:26-34.e1.

16. Papageorgiou SN, Koletsi D, Iliadi A, Peltomaki T, Eliades T (2020) Treatment outcome with orthodontic aligners and fixed appliances: a systematic review with meta-analyses. Eur J Orthod 42:331-343

17. Knierim K, Roberts WE, Hartsfield J Jr. (2006) Assessing treatment outcomes for a graduate orthodontics program: follow-up study for the classes of 2001-2003. Am J Orthod Dentofacial Orthop 130:648-55, 655.e1-3

18. Pinskaya YB, Hsieh TJ, Roberts WE, Hartsfield JK (2004) Comprehensive clinical evaluation as an outcome assessment for a graduate orthodontics program. Am J Orthod Dentofacial Orthop 126:533-543

19. Shim J, Ho KCJ, Shim BC, Metaxas A, Somogyi-Ganss E, Di Sipio R et al (2019) Impact of post-orthodontic dental occlusion on masticatory performance and chewing efficiency. Eur J Orthod. https://doi.org/10.1093/ejo/cjz095

20. Bjering R, Sandvik L, Midtb $\varnothing$ M, Vandevska-Radunovic V (2017) Stability of anterior tooth alignment 10 years out of retention. J Orofac Orthop 78:275-283

21. Bjering R, Vandevska-Radunovic V (2018) Occlusal changes during a 10-year posttreatment period and the effect of fixed retention on anterior tooth alignment. Am J Orthod Dentofacial Orthop $154: 487-494$

22. Angst C, Eliades T, Papageorgiou SN (2020) Stability of occlusal outcome during long-term retention: the time-dependent variation of the American Board of Orthodontics index. Eur J Orthod. https:// doi.org/10.1093/ejo/cjaa004

23. Papageorgiou SN, Höchli D, Eliades T (2017) Outcome assessment of comprehensive fixed appliance treatment: a systematic review with meta-analysis and methodological overview. Korean J Orthod 47:401-413

24. Mousoulea S, Papageorgiou SN, Eliades T (2017) Treatment effects of various prescriptions and techniques for fixed orthodontic appliances : a systematic review. J Orofac Orthop 78:403-414

25. Vandenbroucke JP, von Elm E, Altman DG, Gøtzsche PC, Mulrow CD, Pocock SJ et al (2014) Strengthening the Reporting of Ob- servational Studies in Epidemiology (STROBE): explanation and elaboration. Int J Surg 12:1500-1524

26. Cangialosi TJ, Riolo ML, Owens SE Jr, Dykhouse VJ, Moffitt AH, Grubb JE et al (2004) The ABO discrepancy index: a measure of case complexity. Am J Orthod Dentofacial Orthop 125:270-278

27. Pulfer RM, Drake CT, Maupome G, Eckert GJ, Roberts WE (2009) The association of malocclusion complexity and orthodontic treatment outcomes. Angle Orthod 79:468-472

28. Mislik B, Konstantonis D, Katsadouris A, Eliades T (2016) University clinic and private practice treatment outcomes in Class I extraction and nonextraction patients: A comparative study with the American Board of Orthodontics Objective Grading System. Am J Orthod Dentofacial Orthop 149:253-258

29. Greenland S, Daniel R, Pearce N (2016) Outcome modelling strategies in epidemiology: traditional methods and basic alternatives. Int J Epidemiol 45:565-575

30. Lin LI (1989) A concordance correlation coefficient to evaluate reproducibility. Biometrics 45:255-268

31. Bland JM, Altman DG (1986) Statistical methods for assessing agreement between two methods of clinical measurement. Lancet $1: 307-310$

32. Papageorgiou SN, Tilen R, Vandevska-Radunovic V, Eliades T (2020) Occlusal outcome of orthodontic treatment with pre-adjusted straight-wire and standard Edgewise appliances: a retrospective cohort study [Data set]. Zenodo. https://doi.org/10.5281/ zenodo. 3663626

33. Kattner PF, Schneider BJ (1993) Comparison of Roth appliance and standard edgewise appliance treatment results. Am J Orthod Dentofacial Orthop 103:24-32

34. Soltani M, Saedi B, Mohammadi Z (2012) Outcome of MBT and standard edgewise techniques in treating $\mathrm{Cl}$ I malocclusion. Avicenna J Dent Res 4:61-65

35. Lombardo L, Ficara P, Maltoni I, Moser L, Guarneri MP, Siciliani G (2012) Comparison of the anterior limit of the dentition in patients treated with self-ligating straight-wire, conventional straight-wire and standard edgewise appliances. ISRN Dent 2012:748758

36. Akhoundi MS, Hashem A, Noroozi H (2009) Comparison of occlusal balance contacts in patients treated with standard edgewise and preadjusted straight-wire appliances. World J Orthod 10:216-219

37. Beg Z (2002) Assessment of straightwire vs standard edgewise orthodontic treatment using the Par Index. Master thesis. Oregon Health \& Science University, Portland, Oregon

38. Mavragani M, Vergari A, Selliseth NJ, Bøe OE, Wisth PL (2000) A radiographic comparison of apical root resorption after orthodontic treatment with a standard edgewise and a straightwire edgewise technique. Eur J Orthod 22:665-674

39. Reukers EA, Sanderink GC, Kuijpers-Jagtman AM, van't Hof MA (1989) Radiographic evaluation of apical root resorption with 2 different types of edgewise appliances. Results of a randomized clinical trial. J Orofac Orthop 59:100-109

40. Dal-Re R, Ioannidis JP, Bracken MB, Buffler PA, Chan A-W, Franco EL et al (2014) Making prospective registration of observational research a reality. Sci Transl Med 6:224cm1

41. Papageorgiou SN, Cobourne MT (2018) Data sharing in orthodontic research. J Orthod 45(1):1-3

42. Papageorgiou SN, Xavier GM, Cobourne MT (2015) Basic study design influences the results of orthodontic clinical investigations. J Clin Epidemiol 68:1512-1522 